\title{
Consideration on Wavelet through Chinese Archaian Dialectics
}

\author{
Yuanzhi Wang \\ Geographic Department, Dezhou University \\ Dezhou 253023, Shandong, China
}

Tel: 86-534-898-5841 E-mail: wangyuanzhi1@sina.com.cn

The research is financed by Shandong province office of education.No.S07WZ35

\begin{abstract}
Materialistic philosophy opens out universal law of things evolution. Chinese Yinyang theory is materialistic. Wavelet contain dialectic rule inevitably. As material mathematic technique, wavelet can be given a new look by Chinese archaian dialectics.

Keywords: Dialectical materialism, Yin, Yang, Wavelet

The wavelet analysis is a newly, rapidly developed technique in theses years. It has profound theoretical significance and broad scope of application. Wavelet analysis is known as the microscope of Signal analysis. It has a very good effect in such as geophysical exploration, signal processing, image processing, voice synthesis and segmentation, fault diagnosis, analysis of radar signals.
\end{abstract}

\section{Wavelet theory summary}

On Functional theory, function can be Orthogonally Decomposed on $L^{2}(R)$ space. As the principle of orthogonal decomposition on vector space. for $f(t) \in L^{2}(R)$, Orthonormal Basis $g_{i}(t)$ is present on $L^{2}(R)$

$$
\begin{aligned}
& \text { Where, } t \in R, i=1,2, \ldots, f(t)=\sum_{i=1}^{\infty} f_{i} g_{i}(t) \\
& f_{i}=<f(t), g_{i}(t)>=\int_{-\infty}^{+\infty} f(t) g_{i}(t) d t, i \in z \\
& <g_{k}(t), g_{l}(t)>=\int_{-\infty}^{+\infty} g_{k}(t) g_{l}(t) d t=\delta_{k l}, k, l \in z
\end{aligned}
$$

Eq. (1) is transformation formula of function. Eq. (2) is for Calculating the projection components on basis. Eq. (3) is Orthonormal conditions of basis. Transformation is function conversion in the different basis. When wavelet Bases are constructed, function Transform becomes wavelet transform.

In reality the signals to be dealt are discrete, thus discrete wavelet transform (DWT) becomes the main body of wavelet analysis. The basic framework of discrete wavelet construction is multi-resolution analysis (MRA).

One MRA of $L^{2}(R)$ space has two sub-space: scale space $\left\{V_{j}\right\}_{j \in Z}$ and wavelet space $\left\{W_{j}\right\}_{j \in Z}$. $\left\{\varphi_{j, k}\right\}_{j, k \in Z}$ and $\left\{\psi_{j, k}\right\}_{j, k \in Z}$ are orthogonal of the two Spaces. Signal $f(t) \in L^{2}(R)$ is both orthogonally projectioned on two spaces. 


$$
\begin{aligned}
& f_{V_{j}}=\sum_{k \in z}<f, \varphi_{j, k}>\varphi_{j, k} \\
& f_{W_{j}}=\sum_{k \in z}<f, \psi_{j, k}>\psi_{j, k}
\end{aligned}
$$

The signal $f(t)$ is decomposed on wavelet space:

$$
f(t)=\sum_{j \in z} f_{w_{j}}=\sum_{j \in z} \sum_{k \in z}<f, \psi_{j, k}>\psi_{j, k}
$$

But in practice signals can not be decomposed infinitely and the wavelet coefficients of arbitrary scale can not be seeking out, so signals are decomposed J-j times from scale $2^{-J}$ to $2^{-j}$ :

$$
\begin{aligned}
f(t) & =\sum_{k \in z}<f, \varphi_{J, k}>\varphi_{J, k}(t) \\
& =\sum_{k \in z}<f, \varphi_{j, k}>\varphi_{j, k}(t)+\sum_{j \leq j^{\prime} \leq J} \sum_{k \in z}<f, \psi_{j^{\prime}, k}>\psi_{j^{\prime}, k}(t)
\end{aligned}
$$

Wavelet being relatively simple and easy to understand is Haar wavelet, which is given in 1910 by Haar. It is defined as follows:

$$
\begin{array}{ll}
1, & 0 \leq t<1 / 2 \\
\psi(t)=\quad & 1 / 2 \leq t<1 \\
-1, & \text { Other }
\end{array}
$$

\section{Origins and content of Yin and Yang philosophy}

Yin and yang theory is on an important position in the history of Chinese philosophy. Even the yin and yang theory is not regarded as the core of classical Chinese philosophy, yin and yang philosophy has to be admitted as an integral part of ancient philosophy.

Yin and yang is the product of ancient astronomy, astrology and augury. Shi Xi and Shi He of Yao and Shun times were very experienced in the fields of astronomy, calendar, astrology and augury. They accumulated a great deal of astronomy, calendar, and then summed up the theory of natural philosophy through prone Review. Its origin is religious awareness, so there inevitably are signs of religious superstition. Yin and yang theory as a scientific thinking constantly struggle with religious superstition. The ultimate realization of yin and yang areas to philosophy began in the Zhou Dynasty.

Bo Yangfu of Zhou dynasty as official historian said: The qi of Heaven and earth's order can not be disturbed. If the disorder was disturbed, the National will be chaos. Yang inhibited can not release, and Yin oppressed can not increase, so the earthquake happens. "guoyu-zhouyu shang". Here, The yin and yang are interpreted as "qi of Heaven and Earth ", and are two inter-related, but different from each other basic material of the natural world. It is the firs philosophy concept to yin and yang. Since then, the process of yin and yang aspects to philosophy is greatly speeded up, and they are widely used to explain various natural phenomena. Pre-Qin Taoism understood dialectics In-depth, so they did the largest contribution to the development of the yin and yang school. "Lao Tzu Chapter 42" said: Tao begets One. One begets Two. Two begets Three. Three begets all things. All things carry the yin and embrace the yang. And by breathing together, they live in harmony. "Xi ci" points, "(The reaction 
between) one Yin and one Yang is called Dao". All things consist of two mutual opposite and interdependent sides. The yin and yang is not only qi, and is general property of all things . Chuang Tzu pointed out: The extreme of yin is SuSu. The extreme of yang is Hehe. SuSu comes from the heaven. HeHe comes from the earth. As they integrated mutually, things come into being. "Chuang Tzu.tianzifang". Because of the reconstruction of Taoism, the concept of yin and yang becomes more abstract, more abundant. It is not only a specific material, but a universal attribute; It is not only the primitive of everything, but the reasons for the changes in all things, and is is the two interdependent and confronted Forces of the universe. "Yi zhuan" summed up the results of yin and yang study, enriched and developed the content of the yin and yang, and achieved a leap of yin and yang philosophy. Yin line (-- )and yang line (-) are only mathematical abstraction firstly. The author of "Yizhuan" regulated the original structure -- and - two lines of "Book of Changes" to philosophy, marked them as truly universal philosophy Symbols. The author regarded the contradiction, interdependence, opposition, interaction of yin and yang as a universal law, established dialectics whose core were yin and yang ,and "(The reaction between) one Yin and one Yang is called Dao".

\section{The philosophical connotation of Wavelet theory}

Wavelet analysis is decomposition and reconstruction of signal through wavelet-scale space's orthogonal. Wavelet space and scale space can be corresponding abstractly to the "Yin" and "yang." First, it is reflected that all the signals can be decomposed, and decomposition is built on the scale space and wavelet space as "yin and yang" . As Lao Tzu said: " All things carry the yin and embrace the yang."

It is same as the relationship between the yin and yang that the relationship between scale space and wavelet space is interdependent and mutual antagonistic. The relationship between the scale space and wavelet space as the following:

$$
V_{j+1}=V_{j} \oplus W_{j}
$$

$W_{j}$ is $V_{j}$ 's orthocomplement on space $V_{j+1}$.

On the assumption that $\mathrm{M}$ is subspace of $\mathrm{V}$ space which is $\mathrm{n}$ Dimensional Euclidean space, M's inner-integral to V space is also a Euclidean space. Definition is $M^{\perp}=\{\alpha \in V \mid$ all $\beta \in M$, and $(\alpha, \beta)=0\}$, and $M^{\perp}$ is called orthogonal complement. $M^{\perp}$ is also subspace of $\mathrm{V}$ space. And the following theorem: On the assumption that $\mathrm{M}$ is subspace of $\mathrm{n}$ Dimensional Euclidean space, $V=M \oplus M^{\perp}$. This shows that orthogonal complement is an excellent example which explains interdependence, mutual antagonism, transition in philosophy field.

In addition, "the father wavelet" and "mother wavelet" should be relative to the yin and yang in a sense. Further, 1 and $-1,1$ and 0 in scale function and wavelet function should be relative to yin and yang in the abstract senses.

To some extent, it is mechanical and inappropriate that the wavelet analysis theory should be relative to the "yin and yang" theory. The philosophical thinking in Wavelet analysis is abstract, and it should grasp the wavelet analysis at a deeper level.

\section{References}

A. Cohen and I. Daubechies. (1993). Nonseparable bidimensional wavelet bases. Rev. Mat. Iberoamericana, ,9, 51-137.

Bai, Jing. (2004). On the Nature of "Yin-yang" and its Study in View of Modern Scientific relativity. Journal of Yanshan University (Philosophy and Social Science Edition), 4, 67-69.

Christos, Chrysafis. (2000). Wavelet image compression rate distortion optimizations and complexityreduction. A dissertation for the degree doctor of philosophy.

J.C. Anderson. (1993). A wavelet magnitude analysis theorem. IEEE Transaction on Signal Processing, Special Issue on Wavelet and Signal Processing, December, 41, 3541-3545.

Qiao, Anshui. (2003). An Investigation of the Sources of Yin-Yang Theory. Journal of Yantai University (Philosophy and Social Science Edition), 03, 32-33. 